Mitteilungen der Österreichischen Geographischen Gesellschaft, 158. Jg. (Jahresband), Wien 2016, S. 396-399

\title{
Erich Hillbrand zum 90. Geburtstag
}

\author{
Robert RILL, Wien* \\ mit 1 Abb. im Text
}

Die Familie des Jubilars ist seit den 1470er Jahren im steirischen Salzkammergut nachweisbar und war zumeist im Rahmen des Salzbergbaus tätig. Erich HiLlbrand wurde am 30. April 1926 als Sohn eines Schriftsetzers geboren und besuchte zunächst das Realgymnasium in Wien XVIII, Schopenhauerstraße, das er jedoch unterbrechen musste, da er 1943 als Luftwaffenhelfer eingezogen wurde. Es folgten Arbeits- und Militärdienst, erst bei der Luftwaffe, dann als Panzergrenadier, wobei HILLBRAND auch an der sogenannten „Ardennenoffensive“ teilnehmen musste. Im Februar 1945 geriet er im „Reichswald“ bei Cleve zunächst in kanadische, dann in englische Gefangenschaft. Es folgte französische Kriegsgefangenschaft, bei der er östlich von Beauvais als landwirtschaftlicher Arbeiter auf einer Zuckerrübenplantage eingesetzt war. Deshalb konnte der Schulbesuch erst ab 1946 - nunmehr am Realgymnasium in Bruck an der Mur, denn die 1945 ausgebombte Familie war inzwischen nach Aflenz übersiedelt - fortgesetzt und 1949 mit der Matura beendet werden.

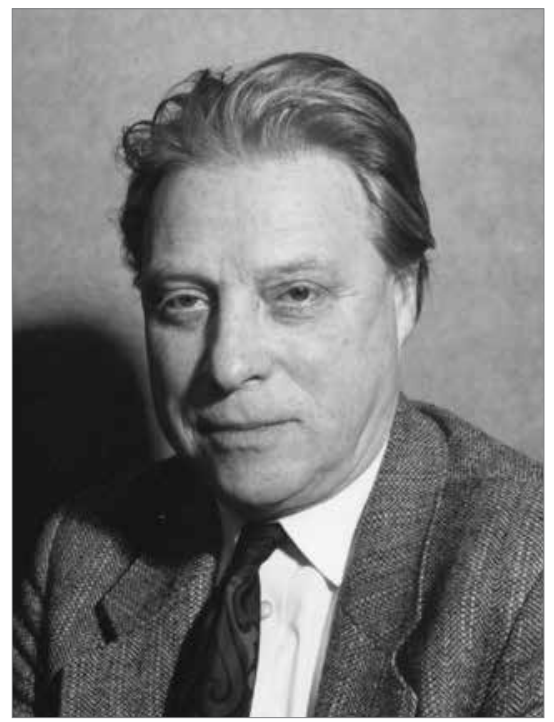

Erich HILLBRAND

* HR Dr. Robert RILl, Österreichisches Staatsarchiv, Kriegsarchiv, Nottendorfer Gasse 2, A-1030 Wien; E-Mail: robert.rill@oesta.gv.at; www.oesta.gv.at 
Im Wintersemester 1949/1950 begann er das Studium der Geschichte und Geographie an der Universität Wien, das er 1953 mit der Promotion zum Doktor der Philosophie bei Alphons LнотsкY (Dissertation: „Das Ungeld in Nieder- und Oberösterreich vom 13. bis zum 19. Jahrhundert mit besonderer Berücksichtigung der Zeit von 1500 bis 1700“) abschloss. Ab 1965 besuchte HILLBRAND den dreijährigen Ausbildungskurs des Instituts für Österreichische Geschichtsforschung (Wien) unter der Leitung von Heinrich FichtenAU, wo er nach Ablegen der Staatsprüfung im Jahr 1968 (Institutsarbeit: „Das Werden der Maximilianeischen Befestigung von Linz“ bei Erich ZöLLNER) die Mitgliedschaft auf Lebenszeit erwarb. Seine Institutskollegen waren unter anderen Reinhard Rudolf Heinisch, Walter Höflechner, Werner KöFler, Lorenz Mikoletzky und Heinrich PuRKarthofer. Parallel zu seinen Studien arbeitete HiLlBRAND ab 1950 auch für Kommissionen der Österreichischen Akademie der Wissenschaften (Historischer Atlas der Alpenländer Österreichs, Österreichisches Biographisches Lexikon, Kommission für Burgenforschung) und ab 1955 für das historische Quellenwerk der Linzer Regesten. Im Jahr 1955 führten ihn seine Studien in das Stiftsarchiv von Kremsmünster, 1956 in das Stadtarchiv Wels und in das Oberösterreichische Landesarchiv in Linz.

Im Jahr 1956 begann er auch seinen Dienst im Kriegsarchiv Wien (Österreichisches Staatsarchiv), wo er als Bestandsgruppenleiter die Karten- und Bildersammlung betreute. 1970 avancierte er zum Archivrat, 1974 zum Archivoberrat, 1987 bei zusätzlicher Übernahme der Direktion der Abteilung „Verkehrsarchiv“ (bis zu dessen Auflösung 1989) zum Hofrat. Von 1989 bis 1991 war er schließlich Direktor des Kriegsarchivs, als welcher er auch öfters den Generaldirektor des Österreichischen Staatsarchivs, Kurt Peball, vertrat. Noch 1992 leitete Hillbrand als Konsulent die Übersiedlung des Kriegsarchivs von der Stiftskaserne in den Neubau in Wien-Erdberg. Nebenbei war HILlBRAND in seiner aktiven Dienstzeit auch als Personalvertreter des Österreichischen Staatsarchivs tätig. Seit 1960 war Hillbrand mit der Historikerin und Prosopographin Friederike HiLLBRAND-GRILL (1931-2015) verheiratet, mit der er in wissenschaftlichen Belangen eng zusammenarbeitete. Im Jahr 1964 wurde die Tochter Edith (verehelichte DoLEISCHI) geboren.

HILlBRAND interessierte sich schon seit seiner frühesten Studentenzeit besonders für Fragestellungen der historischen Geographie, der Kartographiegeschichte, der Entwicklung des Festungswesens und des Städtebaus. Mit seinem Eintritt in das Kriegsarchiv im Österreichischen Staatsarchiv war es ihm als Bestandsgruppenleiter der Karten-, Plan- und Bildersammlung möglich, seine diesbezügliche wissenschaftliche Tätigkeit zu intensivieren, wofür seine zahlreichen Publikationen sowie seine unermüdliche Teilnahme und Vortragstätigkeit bei einschlägigen Kongressen und Konferenzen Zeugnis geben. Darüber hinaus waren ihm über seine aktive Dienstzeit hinaus stets die fachwissenschaftliche Betreuung von Forschern und Archivbenützern sowie die Archivarsausbildung ernste Anliegen, sodass bereits damals, wie auch in verstärktem Maße fortgesetzt unter seiner Direktion, das Kriegsarchiv ein europäisches Zentrum der historischen, topographischen und militärwissenschaftlichen Forschung darstellte.

Seine besonderen Interessen und sein Engagement spiegeln sich auch in zahlreichen ehrenamtlichen Funktionen und Ehrungen wider: So war er 1989 Mitglied der Prüfungskommission für die Staatsprüfung am Institut für Österreichische Geschichtsforschung, im Jahr darauf wurde er zum Mitglied der Commission Internationale d'Histoire militaire ernannt und bemühte sich als solches um eine Arbeitsgemeinschaft von Militärarchiven. Im Jahr 1986 verlieh ihm der Präsident der Polnischen Akademie der Wissenschaften in Krakau [Kraków] in Anerkennung besonderer Verdienste um die Bewahrung der Polonica im Kriegsarchiv und überdurchschnittlicher Unterstützung polnischer Forscher die Jubiläumsmedaille „Pro patria et scientia“. Im Jahr seines Ruhestandsantrittes erhielt HilLBRAND noch das Goldene Ehrenzeichen des Österreichischen Schwarzen Kreuzes in Anerkennung der intensiven Zusammenarbeit bei der Beschaffung archivalischer Unterlagen für die Auffindung von Kriegsgräbern, Soldatenfriedhöfen und personenbezogenen Daten im Allgemeinen. 


\section{Auswahl-Bibliographie (chronologisch geordnet)}

Dissertationen zur Geschichte Wiens 1945-1955. In: Wiener Geschichtsblätter, 11, 71, 1956, S. 1-9. Die Maximilianeische Befestigung von Linz. In: Österreichische Akademie der Wissenschaften. Anzeiger, philosophisch-historische Klasse, 94, 1958, S. 405-420.

Die Kartenbestände des Kriegsarchivs Wien für das Gebiet der Tschechoslowakei, Polens, des Baltikums und der deutschen Ostgebiete. In: Zeitschrift für Ostforschung, 7, 1958, S. 87-97.

Aufbewahrungsorte von Quellen und Literatur zur Südostforschung. In: Wiener Südost-Jahrbuch 1959, S. 168-175.

Drei Denkmale aus dem Ersten Weltkrieg im Raum von Wien. In: Wiener Geschichtsblätter, 15 (75), 1960, S. 208-211.

Die Befestigungen des Bisamberges in den letzten 100 Jahren. In: Museumsverein LANGENZersdorf, Rund um den Bisamberg. Ein Heimatbuch, S. 112-131. Wien 1961.

Der Brückenkopf Wien im Ersten Weltkrieg. In: Mitteilungen des Österreichischen Staatsarchivs, 14, 1961, S. 138-144.

Ein Bildwerk über den bosnischen Streifzug des Prinzen Eugen. In: Forschungs- und KulturstelLe der Österreicher aus dem Donau-, Sudeten- und Karpatenraum, Rat der SüdostdeutSCHEN, SÜDOSTDEUTSCHES KULTURWERK, Eine donauschwäbische Gedenkschrift zum 300. Geburtstag des Prinzen Eugen, S. 80-104. Wien 1963, Typographische Anstalt.

Oberösterreich in Sparrs Donauatlas. In: Mitteilungen des Oberösterreichischen Landesarchivs, 8, 1964, S. 298-307.

Die Einschließung von Linz 1741/42 (= Militärhistorische Schriftenreihe, 15). Wien 1970.

Alte Ansichten und Pläne von Esseg in der Kartensammlung des Kriegsarchivs. In: Mitteilungen des Österreichischen Staatsarchivs, 25, 1972, S. 374-382.

Die Kartenbestände des Kriegsarchivs Wien für das Gebiet der ehemaligen Militärgrenze. In: KrIEgsARCHIV WIEN, Die k.k. Militärgrenze. Beiträge zu ihrer Geschichte (= Schriften des Heeresgeschichtlichen Museums Wien/Militärwissenschaftliches Institut, 6), S. 231-253. Wien 1973.

Die Kartensammlung des Kriegsarchivs Wien. In: Mitteilungen des Österreichischen Staatsarchivs, 28, 1975, S. 183-196.

gemeinsam mit Peter BrouceK und Fritz Vesely: Historischer Atlas zur Zweiten Türkenbelagerung Wien 1683. Wien 1982.

Die Wachau im Kartenbild aus den Beständen des Kriegsarchivs. In: Scrinium, 28, 1983, S. 336342.

Die Gefechte bei Feldkirch 1799 und der Kampf um Vorarlberg bis 1801 (= Militärhistorische Schriftenreihe, 52). Wien 1985.

Die Türme von Linz. Erzherzog Maximilians Festungssystem für die Monarchie. Linz 1985.

gemeinsam mit Peter Broucek, Rainer Egger, Panto Kolev: Katalog. In: Gjuzelev V. (Hrsg.), Die bulgarischen Gebiete und die Bulgaren in den Sammlungen des Kriegsarchivs Wien (16641878), S. 11-62. Wien 1986.

Der Bau der Maximilianeischen Befestigung von Linz in wirtschaftlich-sozialer Sicht. In: Mitteilungen des Österreichischen Staatsarchivs, 39, 1987, S. 31-57.

Walter Wagner 1923-1989. In: Mitteilungen des Österreichischen Staatsarchivs, 41, 1990, S. 446451.

Das Kriegsarchiv als Partner der Festungsforschung. In: Studi trentini di scienze storiche: Sezione 2, 70, 1, 1991, S. 55-67.

gemeinsam mit Friederike HILLBRAND: Ein Lothringer zeichnet die niederösterreichische Donauregion. Ein Beitrag zum Leben und Werk François Nicolas Sparrs. In: Jahrbuch für Landeskunde von Niederösterreich, Neue Folge, 59, 1993, S. 93-123. 
Vom alten ins neue Haus - Die Übersiedlung der Karten- und Bildersammlung des Kriegsarchivs. In: Scrinium 47, 1993, S. 324-330.

Das Fort am Pöstlingberg. Der Linzer Hausberg als militärischer Stützpunkt (= Linzer Werkstattgespräche, 9). Linz 1995.

Pauliny Jakob Josef, Pseudonym Schulz. In: Neue Deutsche Biographie, 20, 2001, S. 126.

Das Kriegsarchiv von 1945 bis zur Jahrtausendwende. In: Mitteilungen des Österreichischen Staatsarchivs, 49, 2001, S. 41-58.

Rainer Egger $\dagger$. In: Mitteilungen des Instituts für Österreichische Geschichtsforschung, 118, 2010, S. 541-542. 